\section{IN THE NEWS}

Word problems

An inability to process

language does not

necessarily affect

mathematical ability, UK

researchers have found.

"This suggests mathematical reasoning can exist without language" (BBC News Online, 15 February 2005).

A team led by Rosemary Varley at the University of

Sheffield made the discovery by studying three patients with severe aphasia - an impairment in the ability to understand and to produce grammatically correct language.

Although the patients understood the words 'lion', 'hunted' and 'man', they were not able to tell the difference between the sentences 'the lion hunted the man' and 'the man hunted the lion'. However, when they were presented with sums such as '52 minus 11 ' and '11 minus 52', which were structured in a similar way, they had no problem with either.

Varley speculates that "It might be possible to use this capacity for mathematical grammar to help the patients find a way to interpret speech and the written word" (news@nature.com, 14 February 2005).

The discovery challenges a commonly held view that language processing is a fundamental skill that is used for related grammatical tasks in the brain, such as certain mathematical ones. "We

have blown that one to pieces, I think" Varley told New Scientist (19 February 2005).

Some have gone even further. According to New Scientist, the study indicates that "mathematical and language skills evolved independently".

However, not everyone agrees. "That does not rule out the notion that both syntax and math abilities derive from common architecture" cognitive psychologist Stanislas Dehaene told Science Now (15 February, 2005)

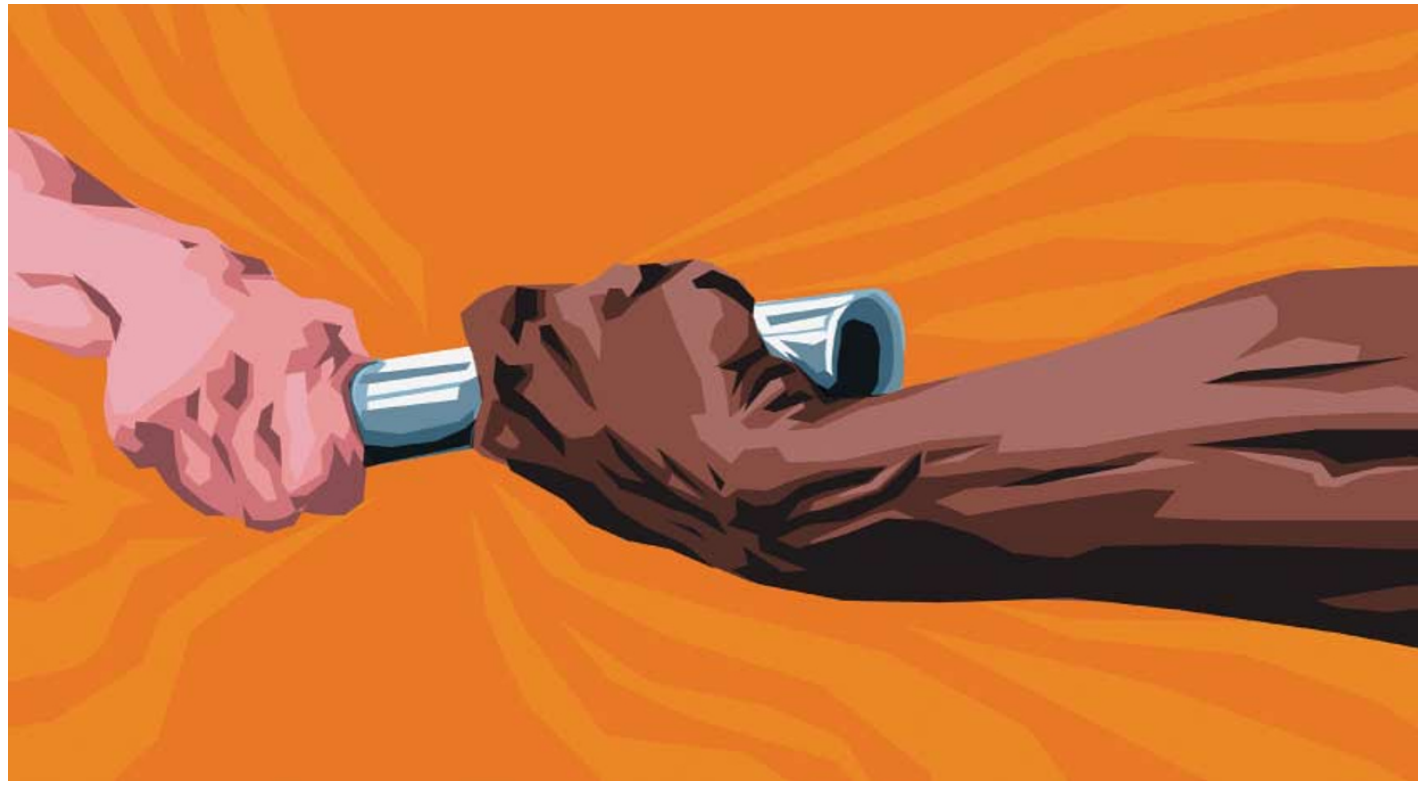

COGNITIVE NEUROSCIENCE

\title{
Grasping intentions
}

Understanding the intentions of others is the basis of social interaction, but the neural mechanisms that underlie this ability remain elusive. Now a paper published in PloS Biology reports the intriguing discovery that mirror neurons, which were thought to be involved in only the execution and recognition of actions, are also important for reading the intentions of those actions.

Mirror neurons were first identified in monkeys. They fire bursts of electrical activity either when a monkey performs object-directed movements, such as grasping and tearing, or when it observes others either another monkey or a human experimenter performing similar movements. Subsequent studies using functional MRI (fMRI) and other techniques have found similar mirror neurons in humans.

In this new study, Iacoboni and colleagues investigated a hunch that mirror neurons might also be involved in detecting the intentions of actions. They proposed that if mirror neurons were only involved in the execution and recognition of actions then their activity would be unaffected by the presence or absence of context.

To test whether this was the case, they presented three types of movie clip - 'action', 'context' and 'intention' - to 23 healthy volunteers and measured their brain activity using fMRI. The action clip showed an empty mug and a hand reaching from off screen to grasp it. There were two versions of the context clip: in the first, a mug of tea, a teapot, a pitcher of cream and a plate of cookies sat neatly on a nondescript surface; in the second, the mug was empty, the pitcher was on its side and a crumpled napkin lay beside scattered cookie crumbs - the remains of an apparently well-enjoyed snack. The intention clip showed the grasping action in both of these contexts, thereby providing the information required to understand the intention - drinking in the first context and cleaning up in the second.

Both the action and intention, but not the context, clips activated the right frontal cortex, which is known to be part of the mirror neuron system in humans. However, the intention clip (grasping in the presence of context) elicited stronger signals than the action clip (grasping alone), which indicates that neurons in this region are concerned not only with the motion but also with the motivation behind it. Intrestingly, the activity of the right frontal cortex also depended on the intention of actions, with the intention of drinking triggering stronger signals than that of cleaning.

The findings challenge the conventional view that the perception of an action and the interpretation of the reason for the execution of that action rely on different mechanisms, and indicate that mirror neurons are involved in both processes. Understanding how the brain reads the intentions of others could not only offer better insights into social interaction, but could also have important implications for the development of effective therapies to treat patients with autism and similar disorders.

Jane Qiu

\section{(2) References and links}

ORIGINAL RESEARCH PAPER lacoboni, M. et al. Grasping the intentions of others with one's own mirror neuron system. PloS Biol. 3, E79 (2005) FURTHER READING Rizzolatti, G. \& Craighero, L. The mirror-neuron system. Annu. Rev. Neurosci. 27, 169-192 (2004)

WEB SITE

Rizzolatti's laboratory: http://www.unipr.it/arpa/mirror/english/staff/rizzolat.htm 